Geistige Eigentumsrechte, was steht für die Entwicklungsländer auf dem spiel?

\title{
Entwicklungszusammenarbeit : für eine echte Partnerschaft zwischen Bund und Privatwirtschaft
}

\author{
Laurent Guye
}

\section{(2) OpenEdition}

\section{Journals}

Electronic version

URL: http://journals.openedition.org/sjep/694

DOI: $10.4000 /$ sjep.694

ISSN: 1663-9677

\section{Publisher}

Institut de hautes études internationales et du développement

\section{Printed version}

Date of publication: 1 avril 1998

Number of pages: 119-125

ISSN: 1660-5926

Electronic reference

Laurent Guye, « Entwicklungszusammenarbeit : für eine echte Partnerschaft zwischen Bund und Privatwirtschaft », Schweizerisches Jahrbuch für Entwicklungspolitik [Online], 17 | 1998, Online erschienen am: 03 August 2012, abgerufen am 08 September 2020. URL : http:// journals.openedition.org/sjep/694; DOI : https://doi.org/10.4000/sjep.694 


\section{ENTWICKLUNGSZUSAMMENARBEIT: FÜR EINE ECHTE PART- NERSCHAFT ZWISCHEN BUND UND PRIVATWIRTSCHAFT}

LAURENT GUYE*

D IE BEDEUTUNG der privatwirtschaftlichen Finanzströme für die Wirtschaftsentwicklung ist heute allgemein anerkannt. Im Zuge der Neuaufteilung der Aufgaben zwischen dem privaten und öffentlichen Sektor, die in den vergangenen Jahren in den Entwicklungsländern selbst stattgefunden hat, der volumenmässigen Stagnation der öffentlichen Hilfe gegenüber den privaten Direktinvestitionen sowie der Überwindung ideologischer Apriorismen sind die Komplementarität der Finanzströme aus öffentlichen und privatwirtschaftlichen Quellen und die Suche nach Synergien zu einem zentralen Thema geworden. Dies war in der Vergangenheit keineswegs immer der Fall, und es ist das Verdienst des schweizerischen Parlaments, seit 1976 die Mobilisierung privater Ressourcen in einem wichtigen Bereich der Entwicklungshilfe als politisches Ziel zu verfolgen. In Artikel 6 des Bundesgesetzes über die internationale Entwicklungszusammenarbeit und humanitäre Hilfe vom 19. März 1976 steht denn auch zu lesen, dass die Entwicklungszusammenarbeit die Form von «Massnahmen zur Förderung des Einsatzes privatwirtschaftlicher Mittel [...], namentlich von Investitionen », annehmen kann. Im Laufe der Jahre sind die Modalitäten zur Erzielung dieses Hebeleffekts weiterentwickelt worden, und dabei haben sich verschiedene Formen der Partnerschaft zwischen dem Bund und dem privaten Sektor herauskristallisiert. Der vorliegende Beitrag vermittelt einen Überblick über diesen Themenkomplex und über die Entwicklung des von widersprüchlichen Erwartungen und Missverständnissen geprägten Dialogs, in dessen Verlauf die Akteure versucht haben, ein Gleichgewicht zwischen öffentlichen und privaten Interessen herzustellen.

\section{MISCHKREDITE: ÖFFENTLICHES RISIKO UND PRIVATE GEWINNE}

Während nahezu 20 Jahren stellten die Mischkredite volumenmässig das wichtigste Instrument der Entwicklungszusammenarbeit dar, welches bei der Partnerschaft mit dem privaten Sektor eingesetzt wurde. Bei den Mischkrediten handelt es sich um Kredite, die je zu einem Teil aus öffentlichen und privaten Geldern bestehen. Die öffentlichen Gelder waren bis 1987 an Konzessionen gebunden und werden seither als Geschenk vergeben, während die privaten Beiträge in Form von Exporthilfekrediten von einem schweizerischen Bankenkonsortium zur Verfügung gestellt werden. Zusammen stellen diese beiden Finanzierungsquellen einen über zehn Jahre rückzahlbaren Kredit zur Verfügung, einschliesslich drei Jahren Zahlungsaufschub. Der Anteil an unentgeltlichen Zuwendungen macht je nach Verschuldungskapazität des Empfängerlan-

* Sektionschef, Investitionsförderung und Mischfinanzierungen, Wirtschaftspolitische Entwicklungszusammenarbeit, BAWI, Bern. 
des 35 - $50 \%$ des Kredits aus. Der Kredit wird der Regierung des Partnerstaates zur Verfügung gestellt und muss im Rahmen von Entwicklungsprojekten, die sowohl den Prioritäten des Empfängerlandes als auch den Kriterien des schweizerischen Gesetzes über die Entwicklungszusammenarbeit entsprechen, für die Einfuhr von Gütern und Dienstleistungen schweizerischer Herkunft verwendet werden, wobei allerdings ein ausländischer Anteil von bis zu $50 \%$ am Importvolumen zugestanden wird. Dieser Punkt führte denn auch bei den Partnerstaaten und bei den Schweizer Exporteuren zu einem ersten Missverständnis. Das Bundesamt für Aussenwirtschaft (BAWI) verwaltete diese Mischkredite wie ein Entwicklungsinstrument und versuchte sich mittels Missionen, die auf die Abklärungen von unabhängigen Beratern folgten, davon zu überzeugen, dass die vorgeschlagenen Projekte den Entwicklungsprioritäten entsprachen und das von Schweizer Seite gemachte Angebot international wettbewerbsfähig war, d.h. aus technischer und wirtschaftlicher Hinsicht geeignet, die Anforderungen ohne Hilfestellung durch Konzessionsfinanzierungen zu erfüllen. Einige andere OECD-Staaten gingen allerdings weit weniger strikt vor, und manche Empfängerländer hielten mit ihrer Ungeduld nicht hinter dem Berg, denn die unabhängigen Gutachten, auf denen die Schweiz bestand, riefen immer wieder zusätzliche Verzögerungen hervor. Andererseits gewannen die Schweizer Exporteure den Eindruck, das BAWI verhalte sich päpstlicher als der Papst und benachteilige sie im Wettbewerb mit ihren Konkurrenten aus den anderen OECD-Staaten.

Trotz allem konnten auf diese Weise zwischen 1977 und 1996 mehr als zwei Milliarden Franken zusammengetragen werden, wovon die öffentliche Hand rund 803 Millionen bestritt. Die wichtigsten Empfängerländer waren in absteigender Reihenfolge China, Ägypten, Indonesien und Indien. Die am meisten begünstigten Sektoren waren die Energiewirtschaft, die Werkzeugmaschinenindustrie, die Mühlen- sowie die Eisen- und Stahlindustrie. Ziel dieses Beitrags kann es aber nicht sein, eine Impaktanalyse dieser Finanzierungsart durchzuführen, es soll deshalb lediglich erwähnt werden, dass die gesteckten Ziele in den meisten Fällen erreicht werden konnten. Die Tatsache, dass die Empfängerländer mindestens die Hälfte der Finanzierung eines Projektes selber bestreiten müssen, stellt prinzipiell eine Garantie dafür dar, dass die ausgewählten Projekte vom Anfang bis zum Schluss seriös betreut werden. Das Scheitern einiger Projekte zeigt jedoch eine Unzulänglichkeit dieses Instruments auf, nämlich das geringe Engagement des schweizerischen privatwirtschaftlichen Partners. Am Projektrisiko ist dieser nämlich nicht beteiligt, denn der Anteil des Bundes ist nicht rückzahlbar, und die Gelder der Banken sind durch die Exportrisikogarantien gedeckt. Sobald die Vereinbarung unter Dach und Fach ist, muss der Exporteur einzig die Verpflichtungen erfüllen, die sich aus den Serviceklauseln ergeben, worauf er sich selbstverständlich neuen Geschäften zuwendet. Kommen jedoch Schwierigkeiten auf das Projekt oder das damit betraute lokale Unternehmen zu, was vor allem bei Unternehmen des öffentlichen Sektors häufig der Fall ist, so muss letzten Endes das BAWI allein mit den Behörden des Empfängerlandes Gegenmassnahmen ausarbeiten, um den langfristigen Erfolg seiner Bemühungen nicht zu gefährden.

Das begrenzte Engagement des privatwirtschaftlichen Sektors bei den Mischfinanzierungen dürfte dazu führen, dass dieses Instrument zugunsten anderer Modalitäten an Bedeutung verlieren wird. Diese Entwicklung dürfte durch zwei andere wichtige Faktoren noch begünstigt werden. 
Der erste Faktor liegt in der progressiven wirtschaftlichen Öffnung der Entwicklungsländer, ihrer wachsenden Zahlungskraft ${ }^{1}$ und der zunehmenden Liberalisierung des Zugangs zu Devisen. Diese Entwicklung erleichtert den Zugang der Wirtschaftssubjekte zu Bankkrediten in harter Währung, und die Tatsache, dass der nichtrückzahlbare Anteil in den Händen der Regierung bleibt und nicht dem Endverbraucher zur Verfügung gestellt wird, vermindert die Attraktivität der Mischfinanzierungen. Ungünstig für diese Finanzierungsart ist aber auch die Tatsache, dass die Erfüllung der vom Geber- und vom Empfängerland gestellten administrativen Bedingungen viel Zeit erfordert.

Der zweite Faktor betrifft die Regeln hinsichtlich der Vergabe von Mischkrediten, die von den OECD-Ländern 1992 vereinbart wurden. Dieses Regelwerk, auch «Helsinki-Paket» genannt, soll die Verzerrungen abschwächen, die duch die kombinierte Verwendung von Hilfsgeldern und Exportkrediten in den Handelsbewegungen hervorgerufen werden, indem es den Anwendungsbereich von Mischfinanzierungen stark einschränkt. Gemäss der wichtigsten Bestimmung dieser Regeln dürfen Mischkredite nur für Projekte gewährt werden, die kommerziell nicht tragfähig sind (d.h. deren konsolidierter Cash-flow während eines Zeitraums von zehn Jahren negativ ist), z.B. für Projekte zur Verbesserung der sozialen Infrastruktur oder Investitionen im Bereich des öffentlichen Verkehrs, die in der Regel erst nach etwa 25 Jahren rentabel werden. Diese Regeln haben sich markant auf jene Sektoren ausgewirkt, die von der Schweiz traditionell durch Mischfinanzierungen gefördert wurden (Energiewirtschaft, Telekommunikation und Industrieanlagen wie Mühlen, Textilfabriken usw.) und haben dazu geführt, dass das jährliche Volumen der für Mischkredite verwendeten Gelder von knapp 80 Millionen Franken vor Inkrafttreten des Helsinki-Paketes in den vergangenen drei Jahren auf weniger als 30 Millionen Franken gesunken ist. In allen anderen OECD-Ländern wurde eine ähnliche Entwicklung verzeichnet, vor allem in Frankreich, Italien und Deutschland, die in der Vergangenheit ihre Exportindustrien weitaus grosszügiger mit Mischkrediten gefördert haben als die Schweiz.

\section{NEUE POLITIK DER ZUSAMMENARBEIT MIT DER PRIVATWIRTSCHAFT: BOTSCHAFT DES BUNDESRATES VOM 29. MAI 1996}

Mit seiner Botschaft vom 29. Mai 1996 zur «Weiterführung der Finanzierung und über die Neuausrichtung von wirtschafts- und handelspolitischen Massnahmen im Rahmen der Entwicklungszusammenarbeit» ersuchte der Bundesrat das Parlament um die Bewilligung eines fünften mehrjährigen Rahmenkredits, der die Finanzierung der Entwicklungszusammenarbeit zwischen 1997 und 2000 sicherstellen sollte. Gleichzeitig präsentierte der Bundesrat die neu erarbeiteten Grundlagen, auf deren Basis diese Gelder verwendet werden sollen. Die Botschaft selbst stützt sich auf folgende Hypothesen:

- Um sich den aufstrebenden Volkswirtschaften anzunähern und ihre Kosten zu vermindern, sieht sich eine wachsende Anzahl von Unternehmen - nicht nur grosse multinationale Konzerne - gezwungen, einen Teil ihrer Produktion in

1. In diesem Zusammenhang geht es nicht um die am wenigsten entwickelten Länder, die für die Schweiz ohnehin nie als Empfänger von Mischfinanzierungen in Frage gekommen sind. 
die Entwicklungsländer zu verlagern. Die oft zitierte Alternative «exportieren oder investieren» existiert nicht, denn wenn die schweizerische Wirtschaft weiterhin in die Entwicklungsländer exportieren will, muss sie dort Investitionen tätigen.

口 Die langfristige Partnerschaft, die entsteht, wenn ein Unternehmen aus einem OECD-Staat z.B. im Rahmen eines Joint-ventures in einem Entwicklungsland investiert, ist besonders im Hinblick auf ihre Nachhaltigkeit (« sustainability ») qualitativ wertvoller als jene, die sich beim ausschliesslichen Export von Gütern ergibt. Da der Profit von der Dauerhaftigkeit des Projekts abhängt, ist das Unternehmen daran interessiert, dass der Technologietransfer, die Ausbildung des ansässigen Personals, die Unterstützung auf Verwaltungsebene usw. auf optimale Weise abgewickelt werden.

a Die Partnerschaft zwischen dem Bund und der Privatwirtschaft muss neu definiert werden und sich künftig auf eine gemeinsame Wahrnehmung der Risiken stützen, d.h. auf eine Aufteilung der Gewinne und der Verluste.

Diese Prämissen erklären die zentrale Bedeutung der Investitionsförderung, die mit den folgenden Instrumenten realisiert werden soll:

๑ Gründung einer "Schweizerischen Gesellschaft für Entwicklungsfinanzierung» (SGE, provisorischer Name) in Zusammenarbeit mit dem privaten Sektor, welche über Beteiligungen, Kredite oder Garantien die Lancierung oder den Ausbau von Joint-ventures in den Entwicklungsländern und in den mittel- und osteuropäischen Staaten unterstützen soll;

- Ausbau der Kontaktanbahnungsstellen, welche Unternehmen aus den Ländern des Südens und des Ostens bei der Suche nach einem Partner behilflich sein sollen. Das Mandat zur Durchführung dieses Programms während des Zeitraums von 1997 bis 2001 wurde nach einer öffentlichen Ausschreibung an ein privates Consulting- und Auditunternehmen vergeben;

- Finanzielle Beteiligung an Studien zur Vorbereitung von Investitionsprojekten. Wird nach Abschluss der Studie die Investition durchgeführt, so muss der vom Bund gewährte Zuschuss zurückgezahlt werden, andernfalls wird dieser in einen nichtrückzahlbaren Beitrag umgewandelt;

- Förderung des Aufbaus und der Bewirtschaftung von privaten Infrastrukturvorhaben nach dem BOO-Modell («build-own-operate») durch Gewährung von Garantien für den Fall, dass sich die rechtlichen Rahmenbedingungen für die Bewirtschaftung des Projekts verändern sollten;

- Förderung der Ausbildungstätigkeit der Privatwirtschaft;

- Beihilfe zum Transfer umweltverträglicher Technologien;

๑ Unterstützung von Finanzintermediären in den Entwicklungsländern (Risikokapital-, Partnerschafts-, Garantie- und Leasingfonds), die den ansässigen Unternehmen Zugang zu langfristigen Finanzierungsmöglichkeiten verschaffen und Joint-ventures fördern sollen.

Selbstverständlich hängt der Erfolg dieser Instrumente von den Rahmenbedingungen in den Entwicklungsländern ab. Das BAWI verfügt über weitere Instrumente, um auf makroökonomischer Ebene zu einem günstigen Klima beizutragen, wie beispielsweise Zahlungsbilanzhilfen oder Entschuldungsmassnahmen, welche im Rahmen der vom Internationalen Währungsfonds und der 
Weltbank geförderten Strukturanpassungsprogramme zum Einsatz kommen. ${ }^{2}$

Diese neuen Instrumente zur Unterstützung der Privatwirtschaft wurden noch zuwenig erprobt - teilweise ist ihre Konzeption noch gar nicht abgeschlossen -, so dass man heute noch keine Aussagen über ihren Nutzen machen kann. Angesichts der Erfahrungen während der Ausarbeitungsphase und der Reaktionen der Privatwirtschaft anlässlich ihrer Präsentation können jedch schon erste Rückschlüsse über das Potential dieser Instrumente, die zu erwartenden Schwierigkeiten und die unterschiedlichen Erwartungen seitens des privaten Sektors gezogen werden. Diese lassen sich wie folgt in fünf Punkten zusammenfassen:

\section{$\square$ Subsidiarität/Komplementarität}

Öffnet der Beitrag des BAWI tatsächlich wie erwartet zusätzliche private Finanzquellen, oder nimmt er deren Platz ein, so dass der Nutzen für die Entwicklungsländer letzten Endes nicht erhöht wird? Diese Frage kann nicht pauschal beantwortet werden: Je weniger attraktiv ein Empfängerland der Privatwirtschaft erscheint, desto mehr muss auf Anreizmechanismen zurückgegriffen werden.

\section{$\square$ Kurzfristig/langfristig}

Die Instrumente, die der Bund der Privatwirtschaft zur Verfügung stellt, sind auf die langfristige Entwicklung der Weltwirtschaft ausgerichtet, in deren Verlauf die Unternehmen zweifellos gezwungen werden, einen bedeutenden Teil ihrer Technologien und ihrer Produktion in die Entwicklungsländer auszulagern. Nur wenn sie sich frühzeitig auf die Globalisierung der internationalen Wirtschaftsbeziehungen einstellt, kann unsere Wirtschaft Arbeitsplätze sichern. Leider sind eine grosse Zahl der privatwirtschaftlichen Partner des Bundes noch immer in einem rein exportorientierten Denken verhaftet. Sie wollen verkaufen anstatt investieren und sind daher weit mehr an den traditionellen Instrumenten zur Exportförderung (wie z.B. Mischfinanzierungen und Substitutionsgarantien) als an den neuen Mechanismen interessiert. Es kann deshalb nicht überraschen, dass die einschneidenden Kürzungen der Mittel für Mischkredite (nur 50 Millionen Franken im neuen Rahmenkredit gegenüber den bisherigen 120 Millionen) nicht besonders wohlwollend aufgenommen wurden.

\section{$\square$ Chancengleichheit}

Der Bund formuliert seine Massnahmen zur Entwicklungszusammenarbeit auf der Basis der Bedürfnisse der Entwicklungsländer. Das entscheidende Kriterium ist der Beitrag, den eine Massnahme zur wirtschaftlichen Entwicklung leisten kann. Verständlicherweise jedoch machen die privatwirtschaftlichen Partner häufig jene Unterstützungsleistungen geltend, die ihren Konkurrenten zur Verfügung stehen, wenn sie sich um Fördermittel des Bundes bewerben ${ }^{3}$. Trüge man solchen Argumenten Rechnung, so würde man sich auf gefährliches Terrain begeben, denn es steht fest, dass mehrere OECD-Staaten eine weitaus interventionistischere Politik betreiben und bei der Exportförderung "grosszügiger»

2. Diese Instrumente können im vorliegenden Beitrag leider nicht vorgestellt werden.

3. Hier ist von einzelnen Unternehmen die Rede, und nicht von den Branchenverbänden der Industrie. Diese sind sich nämlich im klaren darüber, dass die Massnahmen des Bundes im Rahmen des Gesetzes über die internationale Entwicklungszusammenarbeit von 1976 nicht dafür gedacht sind, die Chancen eines Unternehmens bei einer öffentlichen Ausschreibung zu erhöhen. 
vorgehen als die Schweiz. Aber selbst auf die Gefahr hin, seine Partner aus dem privaten Sektor zu enttäuschen, muss sich der Bund mit der Erklärung begnügen, dass das Entwicklungshilfemandat, welches vom Parlament durch die Bewilligung der Rahmenkredite periodisch weitergeführt wird, Vorrang besitzt. In Anbetracht der drastischen Kürzung der Mittel für Mischfinanzierungen konnte jedoch mit der Einrichtung eines Ausgleichsfonds für Finanzierungsbedingungen ein Zugeständnis an die Exportindustrie gemacht werden. Dieses neue, dissuasive Instrument soll Schweizer Unternehmen, welche bei öffentlichen Ausschreibungen aufgrund der vorteilhafteren Finanzierungsbedingungen der Konkurrenz benachteiligt werden, die Möglichkeit bieten, ihr Finanzierungsangebot demjenigen der Konkurrenz anzugleichen. Dieser Ausgleichsfonds verfolgt aber auch ein wichtiges Entwicklungsziel: Indem er die anderen OECD-Länder davon abhält, bei der Exportförderung zu grosszügig zu verfahren, trägt er zur qualitativen Verbesserung der gesamten Hilfeleistungen bei, die die Entwicklungsländer von OECD-Staaten erhalten.

\section{$\square$ Willkür vermeiden}

Der neue Modus der Zusammenarbeit zwischen Bund und Privatwirtschaft bedingt, dass bei den Vertragsabschlüssen und bei der Entscheidungsfindung neue Mechanismen zum Zuge kommen. Bei den Mischkrediten ist der Vertragsabschluss Aufgabe des Empfängerlandes, wobei nach Möglichkeit ein internationales Ausschreibungsverfahren durchgeführt werden sollte. Dieses Verfahren, welches idealerweise sowohl Chancengleichheit für die interessierten Unternehmen als auch ein möglichst gutes Preis-Leistungsverhältnis für das Empfängerland garantiert, eignet sich jedoch nicht unbedingt für die neuen Instrumente, bei denen die Initiative und die Risikobereitschaft des privatwirtschaftlichen Partners im Vordergrund stehen. Das BAWI legt für jedes dieser Instrumente den Anwendungsbereich, die Zielvorgaben, die Mechanismen zur Mobilisierung der privatwirtschaftlichen Ressourcen sowie einige allgemeine Teilnahmebedingungen fest. Anschliessend ist es an der Privatwirtschaft, die gebotenen Möglichkeiten zu nutzen und Zeit und Geld in die Ausarbeitung eines Projektes zu investieren, welches die Unterstützung des BAWI erhalten könnte. Ob diese Unterstützung gewährt wird, hängt von der Übereinstimmung des Projektes mit dem verfolgten Ziel ab, welche vom BAWI analysiert wird. Es ist weder möglich noch wünschenswert, von vornherein eine ausführliche Liste mit Kriterien auszuarbeiten, deren minutiöse Beachtung dem Antragsteller sozusagen ein Anrecht auf Unterstützungsleistungen des Bundes verleihen würde. Der Bund will keine starre Politik verfolgen, sondern er will sich im Gegenteil einen gewissen Spielraum freihalten, der es ihm erlaubt, seine Aktivitäten gemäss der gemachten Erfahrungen neu zu formulieren und auszurichten. Diese Freiheit könnte allerdings den Eindruck vermitteln, dass der Bund Willkür walten lässt. Um dies zu vermeiden, sollen in einer ersten Phase, während der die Einsatzmöglichkeiten dieser neuen Instrumente konkretisiert werden, die eingegangenen konkreten Vorschläge einer kleinen Gruppe von unabhängigen Spezialisten, grösstenteils Vertreter der Privatwirtschaft, unterbreitet werden. Hingegen wird die konsultative Kommission für Aussenwirtschaftspolitik auch weiterhin als kompetentes beratendes Gremium bei der Ausrichtung der Politik und der Programme wirken. 
Die neue Rollenverteilung zwischen Bund und Privatwirtschaft setzt voraus, dass die Initiative zunehmend vom privaten Sektor ausgeht. Wie bereits erwähnt ist es die Aufgabe des BAWI, den Rahmen für die Zusammenarbeit vorzugeben; die Unterbreitung konkreter Projekte jedoch liegt bei der Privatwirtschaft. Dies wirkt sich natürlich auf die Organisation der Arbeit und auf die Beziehungen mit den Regierungen der Empfängerländer aus. Im Extremfall könnte diese Abkehr von der planwirtschaftlichen Logik dazu führen, dass sich der Bund in zu vielen Ländern und in zu vielen Programmen gleichzeitig engagiert, was natürlich negative Folgen für die Effizienz und die Betreuung hätte. Andererseits ist es aber unmöglich geworden, mit den Behörden der Empfängerländer ein festes finanzielles Engagement zu vereinbaren, denn das Volumen der der Finanzströme hängt unter anderem von der Kapazität des privaten Sektors ab, die zur Verfügung gestellten Förderungsmechanismen oder -fazilitäten auszunutzen. Selbstverständlich muss ein Mittelweg zwischen völliger Flexibilität, die zur Verzettelung der Anstrengungen führt, und dem rigiden planwirtschaftlichen Denken gefunden werden, welches in zahlreichen Entwicklungshilfeorganisationen noch immer vorherrscht.

Das BAWI steht am Anfang eines komplexen Experiments, bei dem zahlreiche Partner aus der Schweizer Privatwirtschaft und aus den Entwicklungsländern mit unterschiedlichen Interessen mitwirken. Es liegt in der Natur eines Experiments, dass Neues versucht werden muss, dass Fehler gemacht werden und Korrekturen vorgenommen werden müssen. Die Zusammenarbeit mit dem privatwirtschaftlichen Sektor mag zwar vielversprechend sein, sie ist aber kein Wundermittel. Wie bei zahlreichen klassischen Projekten der Entwicklungszusammenarbeit werden auch hier Probleme hinsichtlich der Qualität, der Nachhaltigkeit und der Eigentumsübertragung auftauchen, wenn auch in etwas anderer Form. Bei der Bewältigung dieser Probleme kann der private Sektor viel von den Entwicklungshilfeorganisationen lernen, und umgekehrt können die Organisationen von den Erfahrungen der Privatwirtschaft profitieren. Wichtig ist, dass der Dialog stattfindet, ohne den keine echte Zusammenarbeit möglich ist. 